\title{
Beam-ion instability
}

\author{
S. Heifets and D. Teytelman \\ Stanford Linear Accelerator Center, Stanford University, Stanford, California 94309, USA
}

(Received 24 November 2004; published 21 June 2005)

\begin{abstract}
In this paper the results of the experimental measurements at Berlin electron storage ring for synchrotron radiation (BESSY-II) are presented. Grow/damp measurements of the transverse coupledbunch instabilities were performed using a multibunch feedback system and a synchronized time-domain bunch motion recorder. The results of these experiments are described and explained by the beam-ion instability. Simplified simulations and revised theory of the instability extended to a nonlinear regime are used for the interpretation of the results.
\end{abstract}

DOI: 10.1103/PhysRevSTAB.8.064402

PACS numbers: 29.27.Bd, 29.20.Dh

\section{INTRODUCTION AND MEASUREMENTS}

Experimental measurements of the coupled-bunch instabilities in the longitudinal and transverse planes are important for both understanding the driving mechanisms and for developing feedback techniques to combat such instabilities. The transverse beam stability at BESSY-II has been investigated experimentally using the longitudinal bunch-by-bunch feedback system as a diagnostic tool. The main motivation for this study was to perfect a measurement technique which uses the downsampled longitudinal feedback (LFB) system as a synchronized data recorder to observe transverse beam motion. Even though the bunch motion is aliased in this process the knowledge of the transverse fractional tune allows one to reliably recover the beam motion in postprocessing [1]. The loop closure of the transverse bunch-by-bunch feedback system was controlled by the LFB in order to generate the grow/ damp transients.

The main relevant BESSY-II parameters, both nominal and the values used for calculations in this paper, are given in Table I.

In this paper, we describe the grow/damp experiments at BESSY-II and suggest an explanation of the results. In the experiments the vertical instability of the beam has been observed. Dipole oscillations of each bunch in the ring were recorded during approximately $16 \mathrm{~ms}$ between the moments when the transverse feedback (FB) has been turned off and on again. A typical result is shown in Fig. 1 where the red and blue colors correspond to the largest and smallest amplitudes, respectively. The measurements were performed below the longitudinal dipole coupled-bunch instability threshold (around $70 \mathrm{~mA}$ ). Thus the beam was longitudinally stable at all times. Measurements presented here were made at beam currents between 50 and $60 \mathrm{~mA}$. The high-frequency amplitude modulation with approximately 16 bucket periodicity is due to the filling unevenness of the individual buckets.

One of the intriguing features of Fig. 1 is the suppression of oscillations. The last may be seen as the tilted blue bands in the figure which correspond to the minimum amplitude of bunch oscillations shifting towards the following bunches almost linearly in time.

Recording of the displacements of each bunch in time allows analysis of the data in terms of the (full ring) coupled-bunch (CB) modes and their time dependence. Results of such an analysis illustrated in Fig. 2 show, first of all, that only few low order CB modes are excited. Because of filling pattern modulation at the 16 bucket periodicity there are sidebands in the modal spectrum separated by $400 / 16=25$ revolution harmonics. The sidebands are more prominent in the 400 bunch fill due to higher bunch filling unevenness.

Time dependence of the five largest CB modes is shown in Fig. 3. This figure clearly shows that the amplitudes of these modes grow exponentially only for a small initial period of time. This period is smaller for the ring fully filled and larger for the fill with a gap in the bunch train. The character of the time variation depends significantly on the length of the gap. Useful information can be extracted by fitting a polynomial to the logarithm of modal amplitudes $\ln x(t)$ in each case. From the polynomial fit one can numerically compute the effective growth rate vs amplitude. In this approach the modal amplitude is represented in the following analytical form:

$$
C_{m}(t)=A_{m} e^{p(t) t}
$$

TABLE I. BESSY-II parameters for transverse instability measurements.

\begin{tabular}{lcc}
\hline \hline \multicolumn{1}{c}{ Parameter } & Nominal & Calculations \\
\hline Energy, GeV & 1.70 & 1.72 \\
Beam current, $\mathrm{mA}$ & 250 & 50 \\
Bunch population & $3.5 \times 10^{9}$ & $0.7 \times 10^{9}$ \\
Circumference $2 \pi R, \mathrm{~m}$ & 240 & 240 \\
Harmonic number $h$ & 400 & 400 \\
Vertical tune, $Q_{y}$ & 6.73 & 6.73 \\
$\sigma_{x / y}, \mu \mathrm{m}$ & $230 / 23$ & $230 / 23$ \\
Beam pipe radius, $b, \mathrm{~cm}$ & $1.1-3.75$ & 1.5 \\
\hline \hline
\end{tabular}




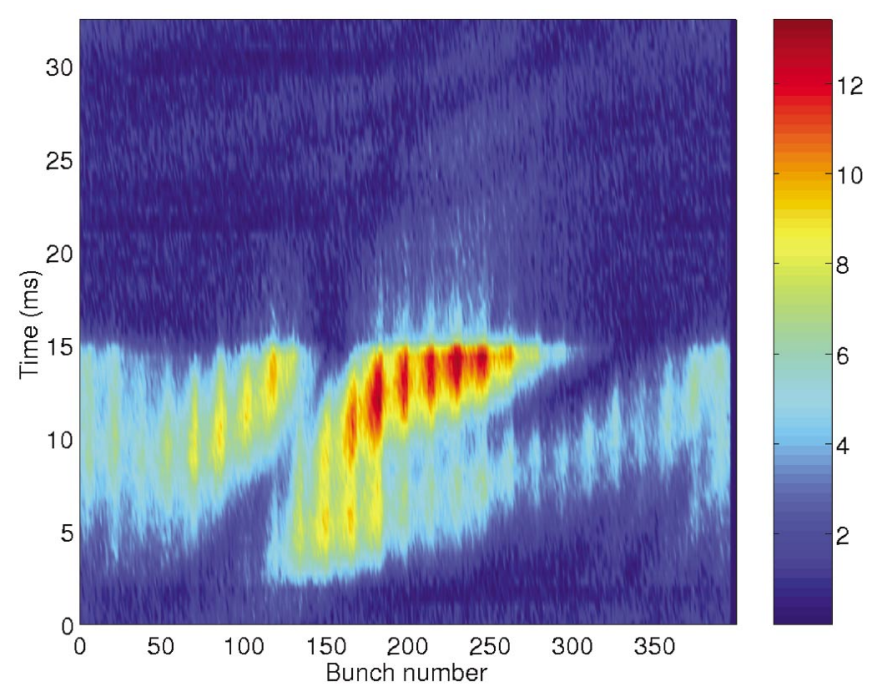

FIG. 1. (Color) Variation of the amplitudes of all bunches with time in the grow/damp experiment at BESSY-II. The transverse feedback system is turned off at $t=0$ and then turned on $16 \mathrm{~ms}$ later. The ring was fully populated with 400 bunches.

where $A_{m}$ is the initial modal amplitude and $p(t)$ is a polynomial defining growth rate variation with time. Such fits are presented in Fig. 4. Note the dramatic difference between a gradual drop in the growth rate with increasing oscillation amplitude for the case of 320 bunches and the fast falloff in the case without a gap. It is interesting to point out that while amplitude of oscillation of mode 399 in Fig. 3(a) grows nearly exponentially, there is a significant frequency shift with the amplitude of oscillation.

Comparing Figs. 3(a) and 3(b) another qualitative difference is evident between the system behavior with and without the gap. Note that without the gap the initial (steady-state) oscillation amplitudes of modes 395-398 are significantly above the noise floor. It is possible to explain such behavior if we assume that instability growth rates have a strong dependence on dipole oscillation amplitude. Then, as the feedback system damps the motion, the growth rates of the relevant modes increase up to the point where they equal the damping provided by the transverse feedback system. Such balance determines the steady-state oscillation amplitudes of the CB modes in question. This hypothesis is further supported by Fig. 4(d) showing a significant increase in instability growth rate at lower oscillation amplitudes.

First we will try to model the observed bunch oscillation amplitude variations as the beating of unstable coherent modes. Such modeling provides further insight into the mechanisms driving the instabilities and allows one to home in on the actual sources of the observed dynamic behavior. a)

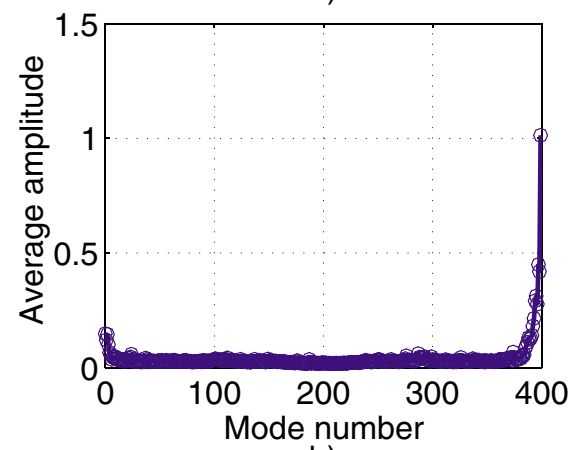

b)

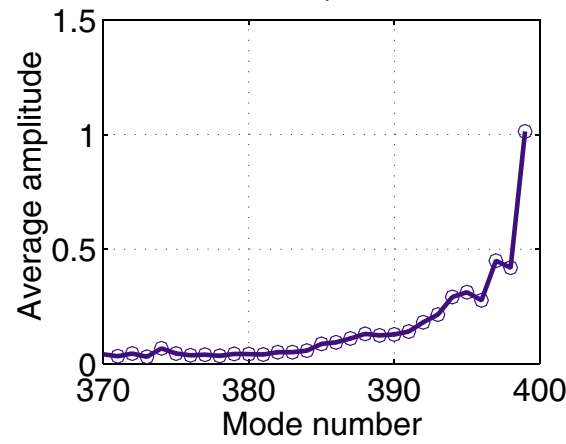

c)

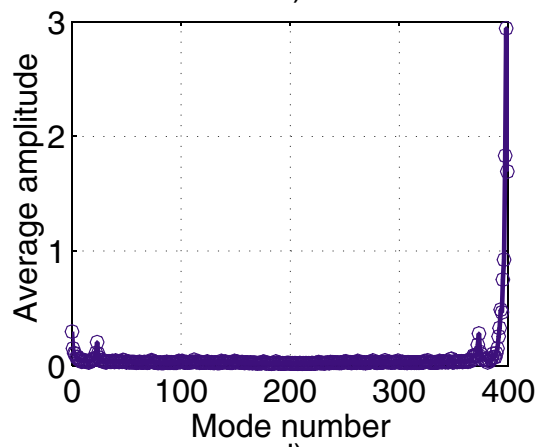

d)

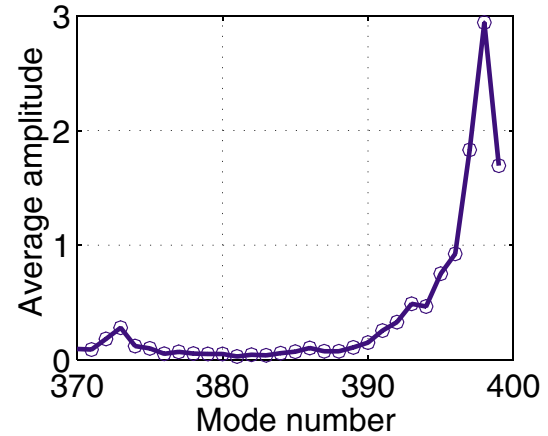

FIG. 2. (Color) Average modal amplitudes (arbitrary units) vs mode number for the growth transient parts of two grow/damp experiments at BESSY-II. (a) 320 bunches in the ring with an 80 bucket (20\%) gap; (b) Plot (a) zoomed in; (c) 400 bunches, no ionclearing gap; (d) Plot (c) zoomed in. 
a)

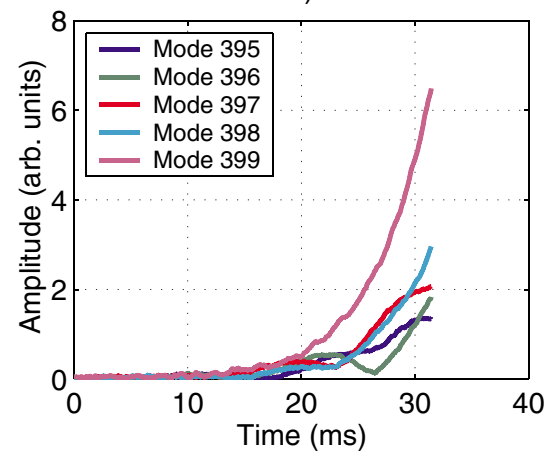

b)

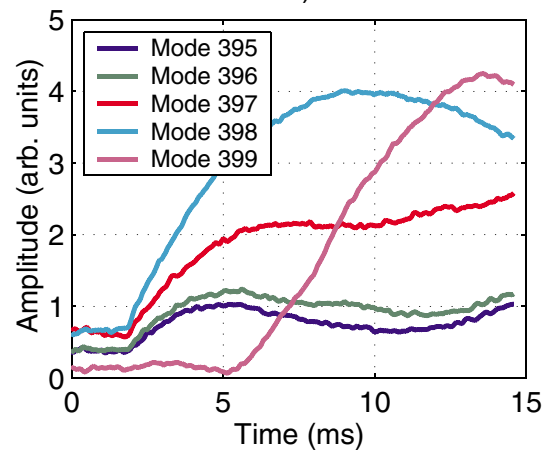

FIG. 3. (Color) Variation of the amplitudes of several CB modes vs time for two grow/damp experiments at BESSY-II. (a) 320 bunches in the ring, 20\% gap; (b) 400 bunches, no ion-clearing gap.

\section{SIMPLE MODEL FOR BUNCH AMPLITUDE VARIATION}

The simplest model explaining the observed variation in bunch oscillation amplitudes can be obtained considering just two coherent coupled-bunch modes with the mode numbers $\mu_{1}$ and $\mu_{2}$, coherent frequencies $\Omega_{1,2}$ and the growth rates $\Gamma_{1,2}$. For simplicity, let us take equal initial amplitudes $a$ for both modes. Then, the vertical displacement of the $N$ th bunch in the train of $h$ equidistant bunches is

a)

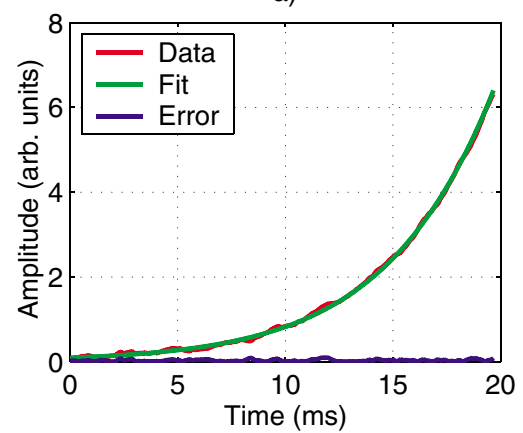

b)

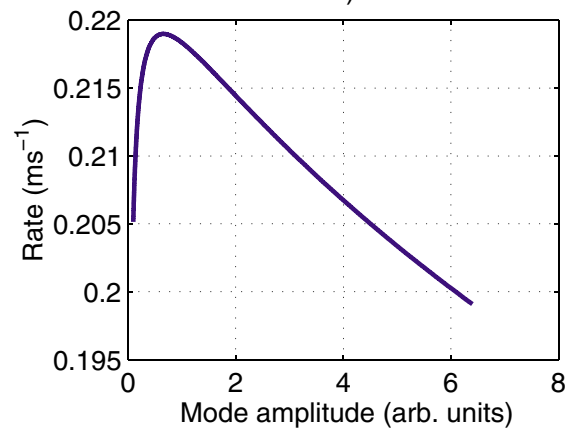

$$
\begin{aligned}
y_{N}(t)= & a\left\{e^{\Gamma_{1} t} \cos \left[\left(\omega_{y}+\Omega_{1}\right) t-2 \pi(N-1) \mu_{1} / h\right]\right. \\
& \left.+e^{\Gamma_{2} t} \cos \left[\left(\omega_{y}+\Omega_{2}\right) t-2 \pi(N-1) \mu_{2} / h\right]\right\},
\end{aligned}
$$

where $\omega_{y}=Q y \omega_{0}$ is the betatron frequency. The amplitude of the bunch varies in time as

$$
\begin{aligned}
A_{N}(t)= & a^{2}\left\{e^{2 \Gamma_{1} t}+e^{2 \Gamma_{2} t}+e^{\left(\Gamma_{1}+\Gamma_{2}\right) t} \cos \left[\left(\Omega_{1}-\Omega_{2}\right) t\right.\right. \\
& \left.\left.-2 \pi N\left(\mu_{1}-\mu_{2}\right) / h\right]\right\}
\end{aligned}
$$
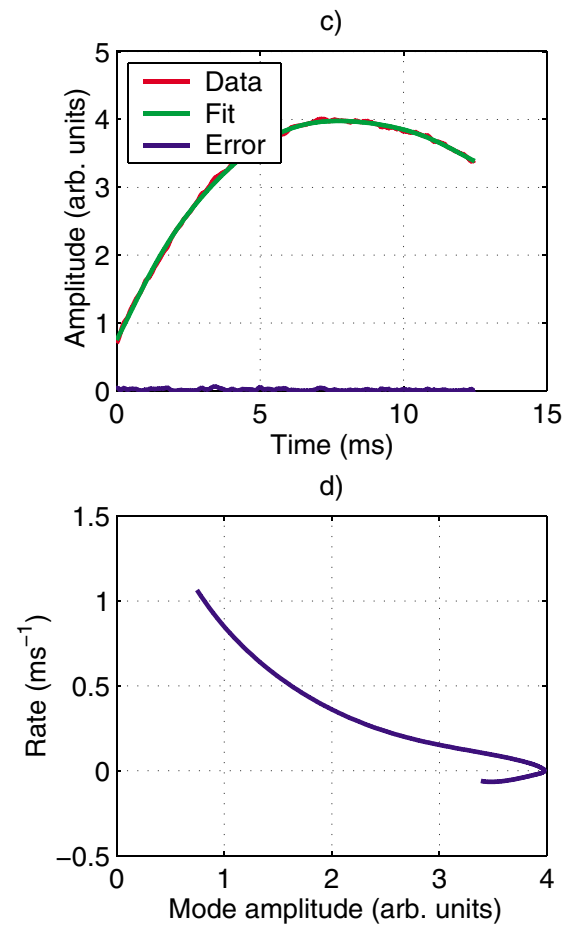

FIG. 4. (Color) Polynomial fits to the logarithm of the amplitude of the dominant CB modes and extracted growth rate dependence on the oscillation amplitude. (a) 320 bunches, third order polynomial fit to the equidistant mode 399; (b) 320 bunches; growth rate of equidistant mode 399 vs amplitude almost constant; (c) 400 bunches, seventh order polynomial fit to mode 398; (d) 400 bunches, fast variation of the growth rate of mode 398 with amplitude. 
The beating is due to the cosine term. First, we notice that this term is independent of the tune $Q_{y}$ and depends on the coherent shift $\Omega$. The minimum amplitude corresponds to the phase equal to multiple of $\pi$. Such minima are separated by

$$
\Delta N=\frac{h}{2}\left(\mu_{1}-\mu_{2}\right)
$$

The beating requires comparable growth rates of two modes $e^{\Gamma_{1} t} \simeq e^{\Gamma_{2} t}$. For an instability with a distinguished strongest mode, both interfering modes have to be close, $\mu_{1}-\mu_{2}= \pm 1$. In this case, two bunches having minimum amplitudes simultaneously would be separated by $h / 2=200$ bunches. For modes with substantial difference in the growth rates, the last term in Eq. (2) becomes negligible with time and the time dependence of the beating may disappear. Still, the amplitude variation along the train may remain provided initial amplitudes of bunches were different. In this case, the depression bands in Fig. 1 would go vertically without slope. Equation (2) shows that the slope of the depression bands is given by the difference in the coherent frequencies of the modes $\Delta \Omega=\mid \Omega_{1}-$ $\Omega_{2} \mid$. A constant phase of oscillation is found for a bunch number $N(t)$ which varies in time with the rate

$$
\frac{d N}{d t}=\frac{h\left(\Omega_{1}-\Omega_{2}\right)}{2 \pi\left(\mu_{1}-\mu_{2}\right)} .
$$

Observation of the slope may provide useful information on the parameters of the coherent modes.

The transient excitation of the beam oscillations $[2,3]$ is another model which would have some features similar to that observed at BESSY in the case of a train with a large gap. Suppose that the first bunch in the train has a small amplitude of betatron oscillations $a$. If there is coupling, each bunch would drive oscillations of the following bunches. If betatron frequencies of all bunches are the same, the amplitude of the second bunch would grow linearly in time until saturation. In the simple daisy-chain model, where a bunch is coupled only to the following bunch,

$$
\frac{d^{2} x_{n}}{d t^{2}}+2 \gamma_{d} \frac{d x_{n}}{d t}+\omega_{b}^{2} x_{n}=\lambda x_{n-1},
$$

the amplitude of the $n$th bunch varies as

$$
a(n, t) \propto \frac{1}{n !}\left(\frac{\lambda t}{2 \omega_{b}}\right)^{n} e^{-\gamma_{d} t} .
$$

The maximum amplitude at the moment $t$ has the bunch with the bunch number $n_{\max }(t)=\Gamma t$, where $\Gamma=\lambda /(2 \omega)$. The amplitude of the bunch with the fastest growth grows exponentially, $a\left(n_{\max }\right)(t) \propto e^{\left(\Gamma-\gamma_{d}\right) t}$. The factor $\Gamma$ gives also the growth rate of coupled-mode instability for the ring without the gap. The position of the bunch with the largest amplitude is shifted along the train with the rate $d n_{\max } / d t=\Gamma$ equal to the growth rate of the instability $\Gamma$.
The growth of the amplitude at BESSY is more complicated with beating and several bands. It is not clear whether BESSY results can be explained as transients in the more elaborated modes where the betatron frequency variation along the train and in time is included.

\section{RESISTIVE WALL AND HEAD-TAIL INSTABILITIES}

The beam instability causing the beating has to have the growth rate fast enough to be observed on the time scale of $16 \mathrm{~ms}$.

Therefore, only the strongest of the conventional transverse wakes, the resistive wall (RW) coherent instability, and the beam-ion instability can be relevant to the observations.

The RW instability is well known and substantially depends on the beam pipe aperture. For this reason, it is difficult to get an accurate estimate of the growth rate of instability for BESSY-II where the aperture has large variations along the ring. Assuming the average $1.5 \mathrm{~cm}$ radius of the aluminum beam pipe, the maximum growth time for 400 uniformly spaced bunches is about $16 \mathrm{~ms}$, equal to the total time of recording in the experiment. However, the aperture in the undulators is $1 \mathrm{~cm}$, and the growth time may be reduced by a factor of 3 and become noticeable in the measurements.

Calculations give the maximum $\Delta \Omega \simeq 100 \mathrm{~Hz}$ for $b=$ $1.5 \mathrm{~cm}$. Hence, for $\Delta \mu=1$, the depression minimum shifts by $\Delta N \simeq 70$ in $10 \mathrm{~ms}$.

The result of calculations where all RW modes are taken into account are shown in Fig. 5. The eigenmodes were

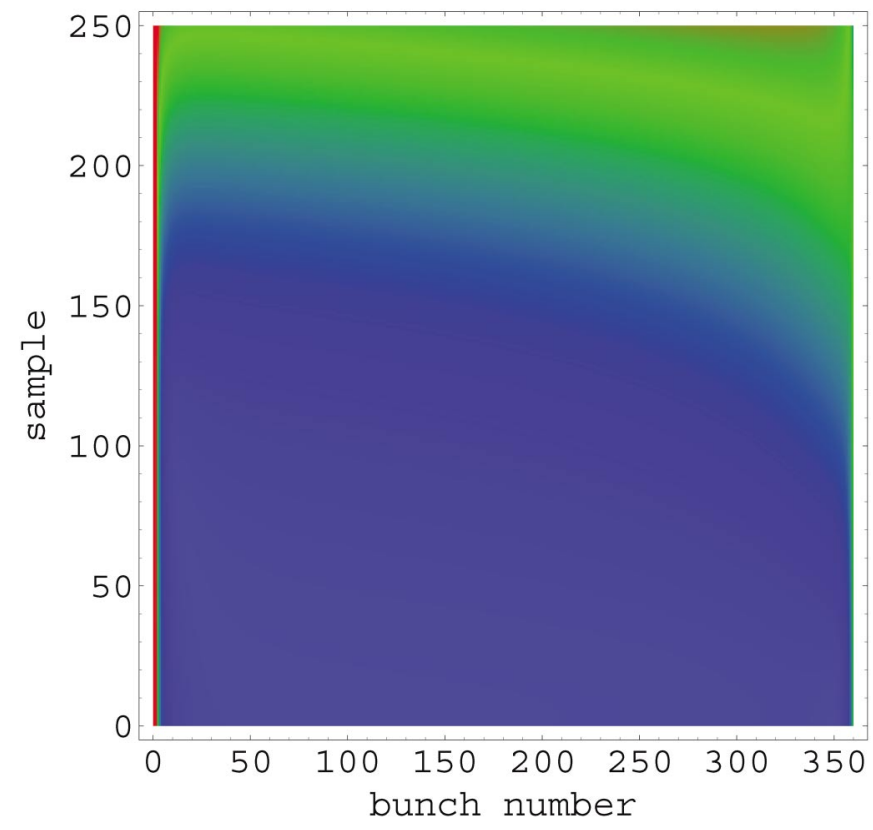

FIG. 5. (Color) Amplitude growth due to RW instability. All 360 modes are taken into account. 
calculated assuming that 360 buckets out of the available 400 are filled leaving a $10 \%$ gap. Initial amplitudes for all modes were taken equal. Although some bunch-to-bunch modulation is visible, the overall variation does not look similar to the experiment.

Several other factors argue as well against the observed instabilities being caused by the resistive wall. As described in the introduction the growth behavior of this instability depends strongly on the presence or absence of the gap. Instabilities driven by the resistive wall impedance should be relatively insensitive to the length of the ion-clearing gap. Secondly, the strong tune shift versus oscillation amplitude observed in a 320 bunch pattern is inconsistent with the resistive wall constant impedance model.

There is frequency shift both for the resistive wall and the beam-ion interactions. The parameter defining the shift is the ratio of the amplitude of oscillations to the beam pipe aperture for the RW case and to the transverse beam rms for ions. Therefore, the frequency shift for ions is substantially stronger for ions.

For all of the above reasons, we consider below only beam-ion instability.

\section{BEAM-ION INSTABILITY}

The beam-ion instability has been studied analytically by many authors $[4,5]$ and numerically [6]. The instability is due to ions which keep memory of the offset of the generating bunch and transfer this information to the following bunches. For large rings, where the revolution frequency is small compared to the ion frequency, the instability becomes the fast ions instability described recently [7]. Let us recall the basic results of the linear theory.

Ions are produced in collisions with the residual gas with the rate

$$
S_{0}=\sigma_{i}^{+} n_{\mathrm{gas}} \frac{N_{b}}{\tau_{b}} .
$$

Here $\sigma_{i}^{+}$is ionization cross section, $\sigma_{i}^{+} \simeq 2$ Mbarn, and $n_{\text {gas }}$ is residual gas density,

$$
n_{\text {gas }}=3.2 \times 10^{7} \frac{p}{\text { nTorr }} \mathrm{cm}^{-3}
$$

at normal temperature. $S_{0} \simeq 10^{8}(\mathrm{cms})^{-1}$ for BESSY-II parameters at 5 nTorr.

Ions with atomic weight $A_{i}$ and initial amplitudes small compared to $\sigma_{x}, \sigma_{y}$, oscillate within the beam with the ion frequency

$$
\Omega_{0}^{2}=\frac{2 N_{b} r_{p} c_{0}^{2}}{A_{i} \sigma_{x} \sigma_{y} s_{b}} .
$$

Here $r_{p}$ is classical proton radius and $c_{0}$ is velocity of light. For BESSY-II parameters, $\Omega_{0} / \omega_{0}=4.2$ and 15.6 for CO and $H_{2}$, respectively. The coasting beam approximation is valid if $\Omega_{0} s_{b} / c_{0} \ll 1$. Otherwise, ions are unstable due to over-kicks from the bunches and cannot be trapped.

For the full ring, ions are accumulated until the space charge of ions becomes comparable with the average density of the beam. The ion density at saturation

$$
n_{\text {sat }}=\frac{2 N_{b}}{\pi s_{b} b^{2}} \ln \left(\frac{b}{\sigma_{x}}\right)
$$

for BESSY-II parameters and 5 nTorr pressure, $n_{\text {sat }} \simeq$ $10^{7} \mathrm{~cm}^{-3}$ and may be reached after approximately 35 turns.

The gap in the train makes most of ions unstable provided the gap length $L_{g}$ is large enough, $\Omega_{0} L_{g} / c_{0}>1$. For BESSY-II and CO, that means $L_{g} / s_{b}>15$. An ion shifts transversely during the first gap by $\Omega_{0}\left(L_{g} / c\right) \sigma_{y}$. The following passes of the gap drive ions toward the wall in a random walk filling the beam pipe uniformly. The estimate of the equilibrium density $n_{\text {gap }}$ can be obtained equalizing diffusive losses at the wall and the production rate. That gives

$$
n_{\text {gap }}=\frac{2 S_{0}}{\omega_{0}\left\langle r^{2}\right\rangle\left(\Omega_{0} L_{g} / c\right)^{2}},
$$

where $\left\langle r^{2}\right\rangle$ is the average distance of ions from the beam squared.

With the 20\% gap and the same 5 nTorr pressure, even for $\left\langle r^{2}\right\rangle=\sigma_{x} \sigma_{y}$ we get $n_{\text {gap }} \simeq 10^{3} \mathrm{~cm}^{-3}$, much smaller than the space-charge limit. This overestimates the cleaning effect of the gap not taking into account that some ions in the ring remain stable even with the long gaps although the number of such ions decreases with $L_{g}$.

Therefore, for the long gap, the ion density is mostly defined by ions generated in one-turn ions with the ion density $n_{i}=S_{0} T_{0} /\left(2 \pi \sigma_{x} \sigma_{y}\right)$, of the order of $3 \times 10^{5} \mathrm{~cm}^{3}$ at the pressure $5 \mathrm{nTorr}$. That sets the lowest limit on the ion density for the last bunch in the bunch train.

The beam-ion instability is usually analyzed in the linearized approximation. Details can be found in the appendix. Oscillation of a bunch at the distance $z$ from the head of the bunch train $y_{c}(t, z)$ in the linear theory is described by the superposition of the CB modes $y_{m}(t, z)=$ $a \sin \left(\mu t-q_{m} z\right)$, where $q_{m}=2 \pi(m-1) / h$ and the coherent frequency $\mu=\omega_{y}+\Delta \omega_{m}$. The growth rate $\Gamma_{m}=$ $\operatorname{Im} \Delta \omega_{m}$ is not zero at the resonance frequency $\Omega(A)$ defined by condition $\omega_{y}+\Omega_{i}(A)=m \omega_{0}$. After averaging over the distribution of ions over amplitudes $A$, the linear growth rate of the mode $q, \Gamma$ is proportional

$$
\Gamma=\frac{\pi r_{e} c_{0}^{2}}{\gamma \omega_{y} \sigma_{y} \sqrt{\sigma_{x}^{2}+\Sigma_{x}^{2}}} \frac{\Omega_{0}}{\Delta \Omega}\left(\frac{d N_{i}}{d s}\right) .
$$

Here, $\gamma$ is the relativistic factor of the beam, $\Sigma_{x}$ is the rms size of the ion distribution, $\Delta \Omega / \Omega_{0}$ is the frequency 
spread of ions. It is large, about $1 / 6.0$ due to strong nonlinearity of the beam-ion potential.

In this limit, the growth time for such a machine as BESSY-II can be as fast as $0.35 \mu$ s for the last bunch even assuming the relatively low one-turn ion density.

Equation (12) shows that the growth rate depends on the ion density. Therefore, the growth rate is higher in the fully filled ring. As a result, the exponential growth lasts longer in the train with a large gap as it was mentioned in Sec. I.

\section{NONLINEAR REGIME OF BEAM-ION INSTABILITY}

The basic equations of the beam-ion instability are reproduced in Sec. 1 of the appendix. In the case of equidistant bunches, motion of the bunch centroid can be described as the superposition of the modes

$$
y_{N}(\tau)=\sum_{n}\left[a_{n} e^{-i Q_{y} \tau+i q_{n} z_{N}}+\text { c.c. }\right],
$$

where $q_{n}=2 \pi n / h, n=0,1, \ldots, n_{b}-1$. (In the case where each $m$-s bucket is filled, the harmonic number $h$ should be replaced by $h / m$.)

$$
y_{N(\tau, s)}(\tau)=\sum_{n}\left[a_{n} e^{-i \nu_{n} \tau-2 \pi i n s /\left(h s_{b}\right)}+\text { c.c. }\right],
$$

where $\nu_{n}=Q_{y}-n$.

That suggests the form of the distance $\zeta_{i}(\tau, s)=$ $Y_{i}(\tau, s)-y_{N(\tau, s)}(\tau)$ between the displacement $Y_{i}$ of an ion at the location $s$ and the offset $y_{N}$ of the bunch $N(\tau, s)$ which happens to be at the location $s$ at the moment $\tau$,

$$
\zeta_{i}(\tau, s)=\sum_{n}\left[A_{i, n} e^{-i \nu_{n} \tau-2 \pi i n s /\left(h s_{b}\right)}+\text { c.c. }\right] .
$$

The system of equations for the amplitudes $a_{n}$ and $A_{i, n}$ is derived in the appendix:

$$
\begin{gathered}
\frac{d A_{i, n}}{d \tau}+\frac{i}{2 \nu_{n}}\left[\Lambda \Phi\left(\left|A_{i, n}\right|^{2}, r\right)-\nu_{n}^{2}\right] A_{i, n}=\frac{i \nu_{n}}{2} a_{n} . \\
\frac{d a_{n}(\tau)}{d \tau}=\frac{i \lambda}{2 Q_{y}} \frac{1}{N_{\text {ion }}} \sum_{i=1}^{N_{\text {ion }}} A_{i, n}(\tau) \Phi\left[\sum_{m}\left|A_{i, m}\right|^{2}, r\right] .
\end{gathered}
$$

In the linear approximation, these equations give well known results, in particular, the growth rate Eq. (12). However, the growth rate defined in the linear regime is high and, usually, the linear regime cannot be observed in the experiment.

In the nonlinear regime the system of equations (16) and (17) can be solved numerically. We used approximation of Eq. (A6) for $\Phi$ and 12 modes $A_{n}, a_{n}, n=, 1, \ldots, 12$ with initial conditions $a_{n}(0)=0.1$ and $A_{n}(0)=0$. The results of calculations shown in Fig. 6 are in good agreement with tracking results. More details are given in the appendix.

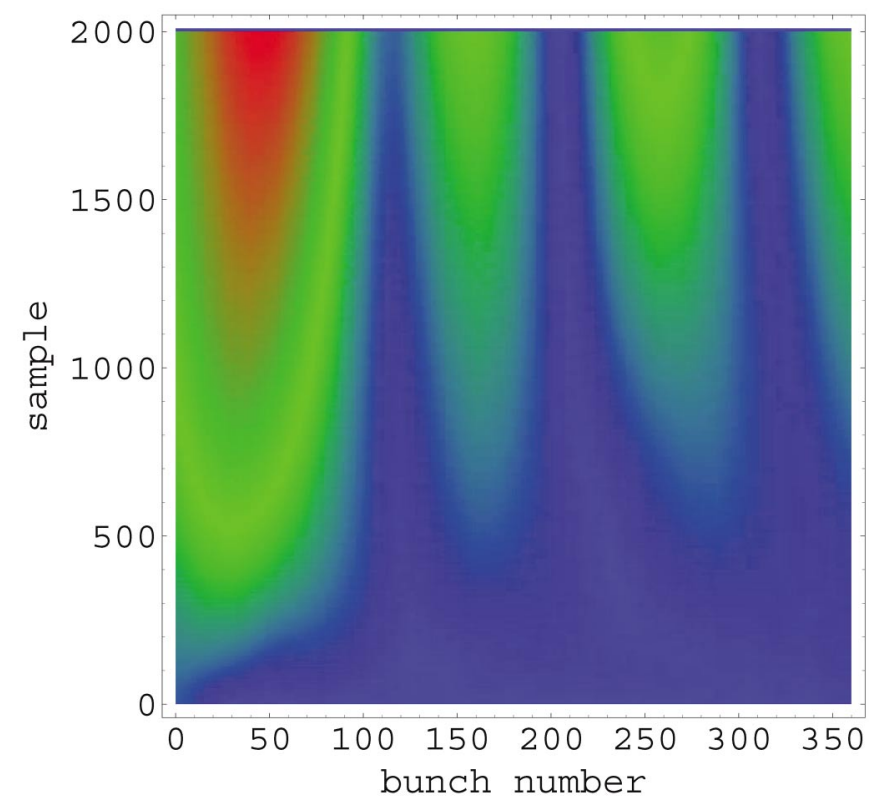

FIG. 6. (Color) Variation of the amplitudes of individual bunches for the beam-ion instability in the simple model of the nonlinear regime. $P=10, J_{0}=10,2 \times 10^{4}$ turns.

\section{SIMULATIONS}

The linear regime, therefore, is too fast to be observed and the nonlinear theory is needed to describe experiments. Some estimates are given in the appendix.

We developed a simple code and used it for simulations. In the code, each bunch is described as a single macroparticle interacting with ions localized in a single slice in the ring. Each bunch passing through the slice generates a macroparticle with the vertical offset equal to the position of a bunch and the rate equal to the production rate of ions in the ring. A bunch gives to and receives kicks from ions existing in the slice. Between bunches, ions are free but get additional kick from the rest of ions to simulate the spacecharge effect. Ions are killed when they reach a certain cutoff distance simulating loss at the beam pipe wall. In addition to the interaction with ions, macroparticles perform betatron oscillations described as a linear map. A resistive wall effect may be added as an additional transform. To do that, the displacement of a bunch is expanded in a sum of the CB modes which are transformed with the known growth rate and then the new coordinate is calculated for the bunch.

The results of simulations are shown in Figs. 7-11. Tracking was carried out for pressure 5 nTorr.

Figures 7 and 8 depict time variation of individual bunches for the train of 320 bunches and for the full ring (400 bunches), respectively. The amplitude value is indicated by color in the same way as in Fig. 1. Although the detail pattern differs from that in the experiment, the qualitative outlook is similar. The suppression bands are clearly visible and their separation is similar to that in Fig. 1. We discuss the difference in the results below. 


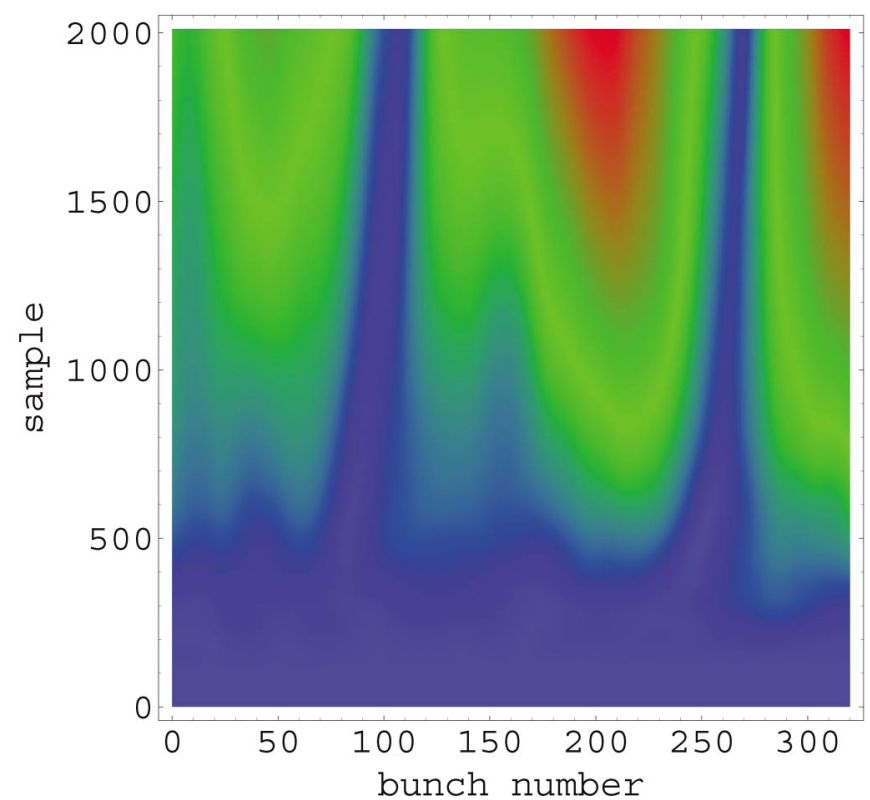

FIG. 7. (Color) Time dependence of the amplitudes of all bunches for a train of 320 bunches in tracking. The horizontal axes gives the bunch number, vertical axes is time sample. Total time of tracking is $2 \times 10^{4}$ turns or $16 \mathrm{~ms}$. The amplitude value is shown in colors; blue and red corresponding to minimum and maximum amplitudes.

Variation in time of the amplitudes of three bunches (in the head, middle, and the tail of the train) is shown in Fig. 9.

Oscillations of individual bunches $y_{n}(t), \quad n=$ $1,2, \ldots, n_{b}$ can be analyzed in terms of the CB modes of

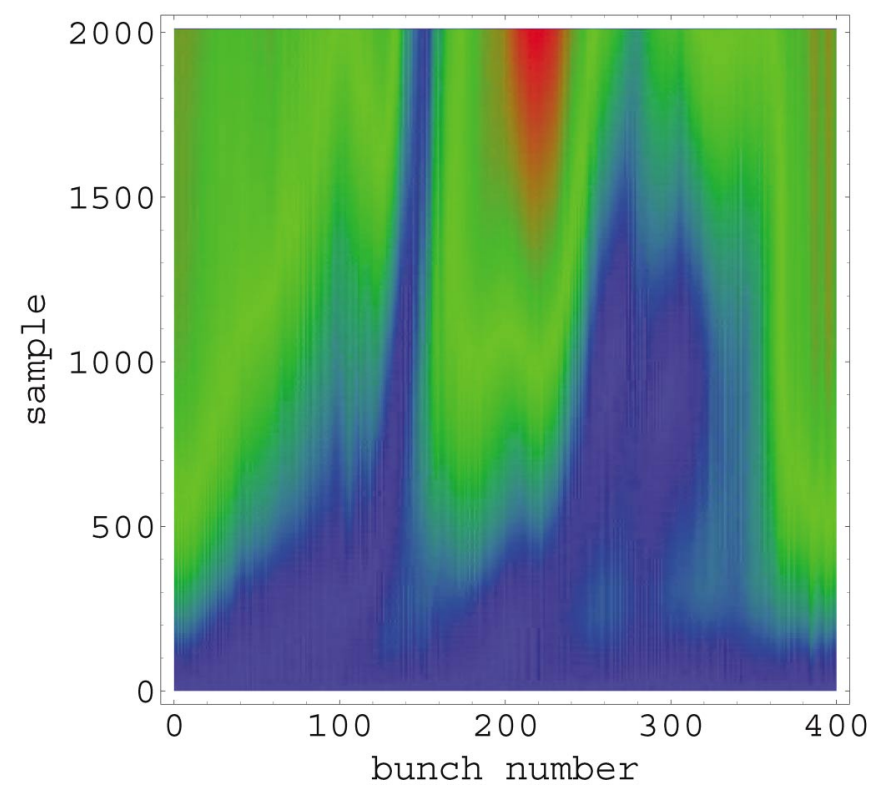

FIG. 8. (Color) The same as in Fig. 7 for 400 bunches (full ring fill).

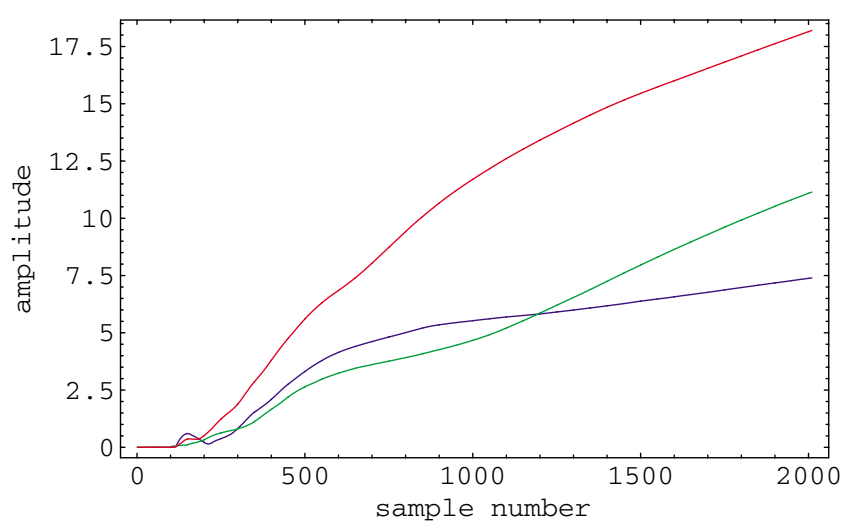

FIG. 9. (Color) Amplitude of three bunches vs time. Blue, green, and red lines correspond to the bunches in the in the head, middle, and the tail of the train of 320 bunches.

the full ring $C_{m}(t), m=1,2, \ldots, h$,

$$
y_{n}(t)=\sum C_{m}(t) e^{2 \pi i(n-1)(m-1) / h} .
$$

Calculated amplitudes of the CB modes are shown in Fig. 10 and are similar to those in Fig. 2. For 320 bunches there are two spectral peaks shown in Fig. 10: at modes 397 and 394. In the case of the uniform fill the peaks are at modes 398 and 396. In Fig. 2(b) a similar multipeak structure is seen for the 320 bunch case with peaks at modes 399 and 397.

Figures 11 and 12 show the time variation of a few strongest modes. The time variation and the time dependence of the amplitudes of individual bunches are not exponential. That shows again that the exponential regime of the linear theory is quickly replaced by much slower growth of the nonlinear regime [8]; see the discussion in the appendix. These results are in agreement with simulations [6].

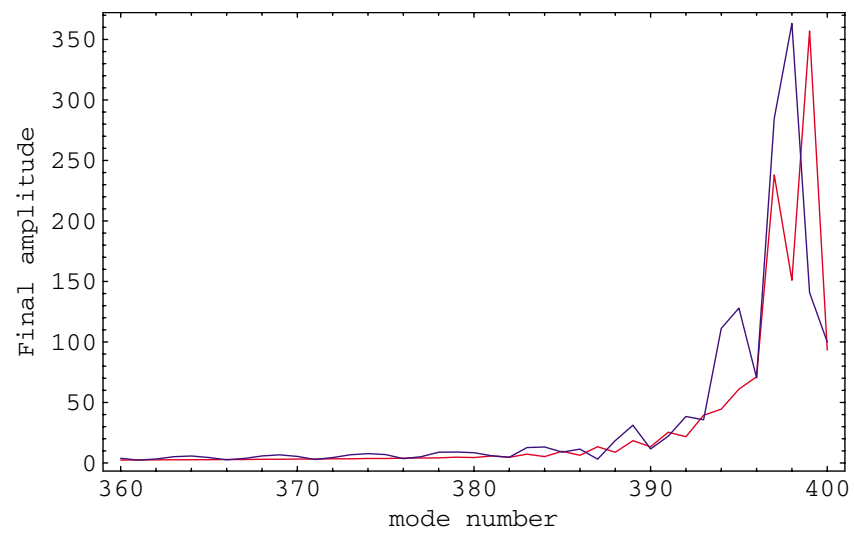

FIG. 10. (Color) Amplitude of CB modes $\left|C_{m}\right|$ vs mode number taken at the end of tracking $(t=16 \mathrm{~ms})$ for the train $n_{b}=320$ (blue) and $n_{b}=400$ (red) bunches. 


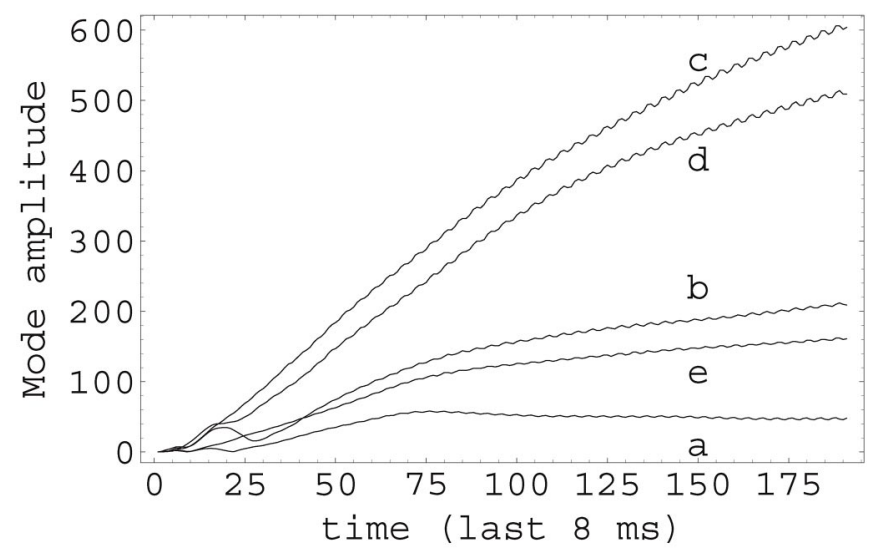

FIG. 11. Time variation of the strongest modes. 320 bunches in the ring.

\section{A. Discussion of simulations}

Experiment and tracking results are quite different for the ring with the large gap (train of 320 bunches) and for the full ring filled (400 bunches). Such a difference can be related to the strong dependence of the ion density on the amplitude of beam oscillations. Figure 13 depicts the time variation of the ion density for the case of 320 bunches in simulations. The beam is artificially maintained stable for the first 1000 turns by turning off the kick from ions to the beam. The ions are produced by each bunch and the ion density growth in a few tens of turns to saturation defined by the space-charge force of accumulated bunches. Quantitatively, the last agrees with the estimate in Eq. (10). After 1000 turns, the kicks to the beam are turned on and the amplitude of the beam oscillations start to grow.

That provides an additional mechanism for ion loss, first, producing ions at larger distances from the beam axes and, second, driving ions to the wall due to the beam-ion interaction. As a result, the density of the ions drops with time although beam amplitude continues to grow.

The strong dependence of the growth rate on the amplitude of the oscillations mentioned in the introduction is the result of the dependence of the growth rate on the ion

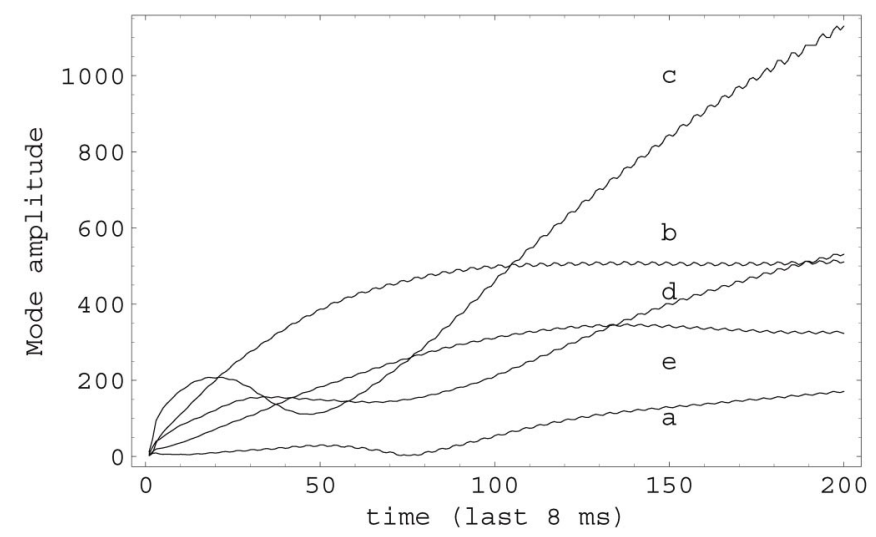

FIG. 12. The same as in Fig. 11 for the train of 400 bunches.

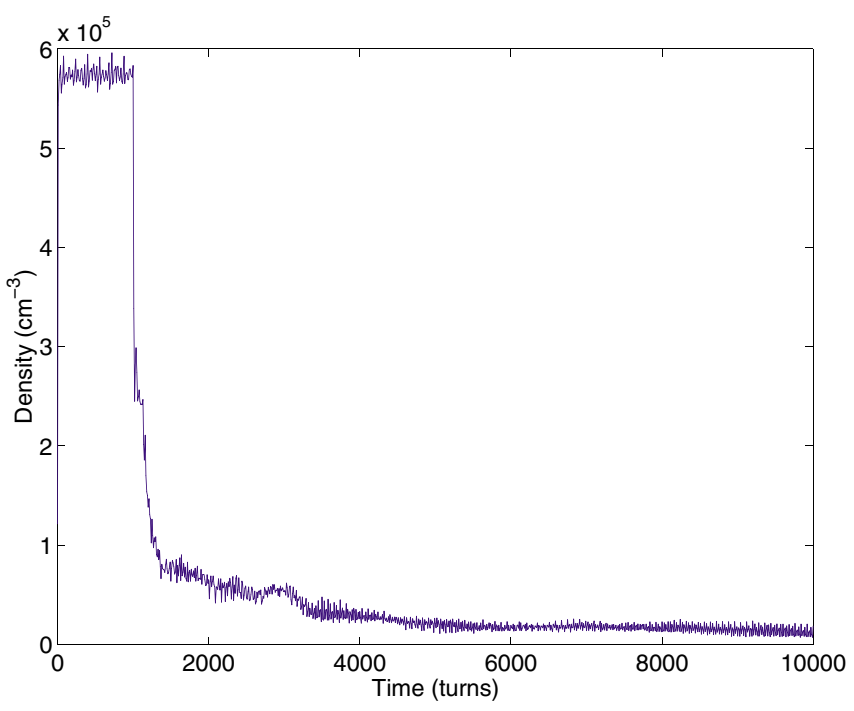

FIG. 13. (Color) Variation of the ion density with time for the train of 320 bunches. The density grows and saturates while the beam is kept stable for the first 1000 turns and then drops with the growth of the beam amplitude.

density which goes down while the amplitude increases. On top of that, the ion distribution becomes wider and flatter. The superposition of the strongest unstable modes generates a complex interference pattern recorded in the experiment.

It should be also true that, in the experiments, the fluctuations of the cloud may be stronger in the case of 400 bunches than in the case of the long ion gaps due to a larger density of the ion cloud. Therefore, the initial amplitude of the beam oscillations when the feedback is turned off can be larger and the instability starts already in the nonlinear regime. That may explain the results shown in Fig. 3(b).

Comparing the experiment with the results of tracking, it is worth noting that the tracking results are quite sensitive to the choice of parameters such as vacuum pressure, transverse rms $\sigma_{x, y}$ of the beam, and the cutoff distance. Some of these parameters are unknown (such as pressure) or may in reality vary in time ( $\operatorname{such}$ as $\sigma_{x, y}$ ). The choice of other parameters (such as the cutoff amplitude, number of ion slices per ring, and variation of the ion frequency around the ring due to variation of the betatron functions) affects the time of simulations and may be prohibiting even in the simplified model we use in simulations. It is also worthwhile to mention that to simulate the grow/damp conditions, we keep beam stable suppressing kicks to the bunches for some number of turns while building the ion density to saturation. Such simulations do not quite reproduce experimental conditions where the turned on transverse feedback does not necessarily mean the zero initial amplitude of the beam oscillations.

Nevertheless, the simulations display the main features of the experimental results. We can conclude that the experiment can be explained by the beam-ion instability. 
Results confirm that the experimentally observable instability corresponds to the nonlinear regime where the density of ions has to be defined in a self-consistent way rather than taken as a constant from separate simulations with a stable beam. The amplitude depression in the train and the split of the amplitude spectrum of CB modes are related to the coherent tune shift and the variation of the ion density with time. More sophisticated simulations may give quantitative prediction in this respect although a comparison with experiment requires more diagnostics on the vacuum pressure in the ring and time variation of the beam rms dimensions.

As it was mentioned in the first section, the exponential growth takes place only for a limited period of time. The period is smaller in the fully filled ring than in the case of a bunch train with a gap. We also noted that the amplitude of oscillations is significantly above the noise floor level if the beam has no gap. Both effects can be explained qualitatively by the interaction with ions. The gap substantially reduces initial density of ions and, before the feedback is turned off, ions have relatively a weak impact on the beam. The linear exponential regime takes place starting with small initial beam amplitudes and continues until the amplitude of the beam oscillations is built up. In the case of a fully filled ring, the initial density of ions and noise on the beam are large. After the feedback is turned off, the growth starts with larger amplitudes and the linear regime is restricted in time.

The interaction with ions can also explain why the strongest mode number is higher without the gap. The strongest mode in the beam-ion instability is the mode shifted up from the betatron frequency by the ion frequency. The latter is defined by the ion amplitude where the ion distribution is maximum. With the large gap, ions are unstable and most of them are one-turn ions with small amplitudes corresponding to a higher ion frequency. For the full ring, most of ions have large amplitudes, lower frequency, and the strongest mode in the beam spectrum closer to the betatron line than in the case of a large gap.

\section{CONCLUSIONS}

The experimental results obtained in the grow/damp experiment at BESSY-II show an intriguing interference pattern and nonexponential dependence of the amplitudes on time. Understanding of these results could provide a new method of diagnostic of the beam parameters. We analyze coherent instabilities looking for a possible explanation of the experimental results. We found that only resistive wall and beam-ion instabilities may be responsible for the results. Further analysis shows that the resistive wall instability is too weak and can be ignored leaving the beam-ion instability as the only plausible candidate. We carried out simplified simulations of the instability whose results were qualitatively similar to the experimental results. In the analysis of results, we reproduce the known results of the linear theory of the instability and developed an approach which allows us to analyze the nonlinear regime of the instability.

Simulations are sensitive to the not very well known parameters such as the shape of the distribution of ions and the pressure of the residual gas. Although we were unable to reproduce the interference pattern exactly, this is not surprising given such a sensitivity to unknown parameters. We believe that results of the paper confirm the beam-ion instability as the source of the interference pattern. Analysis presented in the paper shows, however, that for quantitative comparison more detailed simulations and additional experimental information on the pressure and beam rms size are needed.

\section{ACKNOWLEDGMENTS}

We would like to thank Shaukat Khan of BESSY-II for setting up and participating in the experimental transverse instability measurements presented in this paper. His insightful comments and help with defining BESSY-II accelerator parameters were crucial for the proper data analysis and dynamics simulations. This work was supported by Department of Energy Contract No. DE-AC03-76SF00515.

\section{APPENDIX}

\section{Basic equations}

Let us consider a train of bunches with the transverse rms dimensions $\sigma_{x}, \sigma_{y}, \sigma_{x} \gg \sigma_{y}$, the number of particles $N_{b}$ per bunch, and bunch spacing $s_{b}=c \tau_{b}$. The $n$th bunch is at distances $z_{N}=(N-1) s_{b}, N=1,2, \ldots, n_{b}$ from the head of the train. The position of the $N$ th bunch in the ring is $s_{N}(t)=\left(c t-z_{N}\right) \bmod (2 \pi R)$. Ions at the location $s$ around the ring are described by the coordinates $X, Y$ and have a linear density of $d N_{i} / d s$. We use the dimensionless time $\tau=\omega_{0} t$, vertical $y(\tau), Y(\tau, s)$, and horizontal coordinates $x(\tau), X(\tau, s)$ of the bunches and ions, respectively, measured in units of the bunch rms $\sigma_{y}$ and $\sigma_{x}$.

The beam-ion interaction is just electrostatic Coulomb force. At the moment $t$, ions interact with the bunch numbered $N(\tau, s)=1+\left(c \tau / \omega_{0}-s\right) / s_{b}$. To simplify consideration, we assume that ions are independent of the time Gaussian distribution in $X$ with the average $\langle X\rangle=$ 0 and the rms $\Sigma_{x}$ reducing the problem to the 1D case. We also assume a Gaussian distribution for the beam in the vertical plane with the bunch centroid at $y_{N}(t)$.

The equation of motion for the bunch centroid $y_{N}$ of the $N$ th bunch is obtained by averaging the force over the ion distribution in $X$ and the bunch distribution

$$
\begin{aligned}
\frac{d^{2} y_{N}(\tau)}{d \tau^{2}}+Q_{y}^{2} y_{N}= & -\lambda \int d Y \rho_{i}\left[Y, \tau, s_{N}(\tau)\right]\left[y_{N}(\tau)\right. \\
& -Y] \Phi\left[\frac{y_{N}(t)-Y}{\sqrt{2}}, r\right] .
\end{aligned}
$$


Here $\rho_{i}(Y, \tau, s)$ is normalized to one distribution function of ions at the location $s$ in the ring, $\int d y \rho_{i}(y, \tau, s)=1$, $s_{N}(\tau)=c \tau / \omega_{0}-z_{N}$,

$$
r=\frac{\sigma_{y}}{\sqrt{\sigma_{x}^{2}+\Sigma_{x}^{2}}}, \quad \lambda=\frac{2 r_{e} c_{0}^{2}}{\gamma \omega_{0}^{2} \sigma_{y}\left(\sigma_{x}+\sigma_{y}\right)}\left(\frac{d N_{i}}{d s}\right),
$$

and

$$
\begin{aligned}
\Phi\left(\Delta^{2}, r\right)= & \frac{1+r}{2} \int_{0}^{\infty} \frac{d \xi}{(1+\xi)^{3 / 2} \sqrt{r^{2}+\xi}} e^{-[\xi /(1+\xi)] \Delta^{2}}, \\
= & \sqrt{\frac{\pi}{4 \Delta^{2}}\left(\frac{1+r}{1-r}\right)} e^{r^{2} \Delta^{2} / 1-r^{2}}\left\{\operatorname{Erf}\left[\sqrt{\frac{\Delta^{2}}{1-r^{2}}}\right]\right. \\
& \left.-\operatorname{Erf}\left[\sqrt{\frac{\Delta^{2} r^{2}}{1-r^{2}}}\right]\right\} .
\end{aligned}
$$

Equation of motion for an ion with atomic number $A$ is defined by the Coulomb interaction with passing bunches. In the $1 \mathrm{D}$ model, the interaction is

$$
\begin{aligned}
\frac{d^{2} Y_{i}(\tau, s)}{d \tau^{2}}= & -\Lambda_{0} \sum_{k} \delta[\tau \\
& \left.-\frac{\omega_{0} s_{b}}{c_{0}} k\right] \zeta_{i}(\tau, s) \Phi\left[\frac{\zeta_{i}^{2}(\tau, s)}{2}, r\right],
\end{aligned}
$$

where $\zeta_{i}(\tau, s)=Y_{i}(\tau, s)-y_{N(\tau, s)}(\tau)$. The parameter

$$
\Lambda_{0}=\frac{2 N_{b} r_{p} c_{0}}{A \omega_{0} \sigma_{x} \sigma_{y}} \frac{\Sigma_{x}}{(1+r) \Sigma_{x, t}},
$$

where $\Sigma_{x, t}=\sqrt{\sigma_{x}^{2}+\Sigma_{x}^{2}}$.

The sum over $\delta$ functions can be expanded over the time harmonics. For small bunch spacing, only zero harmonics can be retained. Equation (A4) written in terms of $\zeta(\tau, s)$ takes the form

$$
\frac{d^{2} \zeta_{i}(\tau, s)}{d \tau^{2}}+\Lambda \zeta_{i}(\tau, s) \Phi\left[\frac{\zeta_{i}^{2}(\tau, s)}{2}, r\right]=-\frac{d^{2}}{d \tau^{2}} y_{N(\tau, s)}(\tau)
$$

Here

$$
\Lambda=\frac{c_{0}}{\omega_{0} s_{b}} \Lambda_{0}=\left(\frac{\Omega_{0}}{\omega_{0}}\right)^{2}\left(\frac{2 \Sigma_{x}}{(1+r) \Sigma_{x, t}}\right) .
$$

A good approximation for $\Phi\left(\Delta^{2}, r\right)$ can be written as

$$
\Phi\left(\Delta^{2}, r\right)=\frac{1}{1+\kappa \Delta^{2}},
$$

where $\kappa=(1 / 3)(1+2 r) /(1+r)$.

In the extreme case of large $r \Delta \gg 1, \Phi \simeq=$ $(1+r) /\left(2 r \Delta^{2}\right)$.

In the case of equidistant bunches, motion of the bunch centroid can be described as the superposition of the modes

$$
y_{N}(\tau)=\sum_{n}\left[a_{n} e^{-i Q_{y} \tau+i q_{n} z_{N}}+\text { c.c. }\right],
$$

where $q_{n}=2 \pi n / h, n=0,1, \ldots, n_{b}-1$. (In the case where each $m$-s bucket is filled, the harmonic number $h$ should be replaced by $h / m$.) Then, the right-hand-side in Eq. (A6) is proportional to

$$
y_{N(\tau, s)}(\tau)=\sum_{n}\left[a_{n} e^{-i \nu_{n} \tau-2 \pi i n s /\left(h s_{b}\right)}+\text { c.c. }\right]
$$

where $\nu_{n}=Q_{y}-n$.

That suggests the form of $\zeta_{i}(\tau, s)$,

$$
\zeta_{i}(\tau, s)=\sum_{n}\left[A_{i, n} e^{-i \nu_{n} \tau-2 \pi i n s /\left(h s_{b}\right)}+\text { c.c. }\right] .
$$

Let us replace $\zeta_{i}(\tau, s)^{2}$ in the argument of $\Phi\left\{\left[\zeta^{2}(\tau, s) / 2\right], r\right\}$ by the average value

$$
\zeta_{i}^{2}(\tau, s) \simeq\left\langle\zeta^{2}(\tau, s)\right\rangle=2 \sum_{n}\left|A_{i, n}\right|^{2} .
$$

With the additional condition $A_{i, n}^{\prime} e^{-i \nu_{n} \tau+2 \pi n s /\left(h s_{b}\right)}+$ c.c. $=0$, Eq. (A6) for ions is transformed to

$$
\frac{d A_{i, n}}{d \tau}+\frac{i}{2 \nu_{n}}\left[\Lambda \Phi\left(\left|A_{i, n}\right|^{2}, r\right)-\nu_{n}^{2}\right] A_{i, n}=\frac{i \nu_{n}}{2} a_{n} .
$$

Let us rewrite Eq. (A1) as the sum over all $N_{i}$ ions at the location $s$ :

$$
\frac{d^{2} y_{N}(\tau)}{d \tau^{2}}+Q_{y}^{2} y_{N}=\frac{\lambda}{N_{i}} \sum_{i=1}^{N_{i}} \eta_{i, N}(\tau) \Phi\left[\frac{\eta_{i, N}^{2}(\tau)}{2}, r\right],
$$

where $\eta_{i, N}(\tau)=Y_{i}\left[\tau, s_{N}(\tau)\right]-y_{N}(\tau)$. Using identity $\left.N(\tau, s)\right|_{s=s_{N}(\tau)}=N$, and the definition of $\zeta_{i}(\tau, s)$, it is easy to see that $\eta_{i, N}(\tau)=\zeta_{i}\left[\tau, s_{N}(\tau)\right]$. Then, Eq. (A11) gives

$$
\eta_{i, N}(\tau)=\sum_{n}\left[A_{i, n} e^{-i Q_{y} \tau+i q_{n} z_{N}}+\text { c.c. }\right] .
$$

Using the additional condition $a_{n}^{\prime} e^{-i Q_{y} \tau+i q_{n} z_{N}}+$ c.c. $=$ 0 , we get from Eq. (A14) equation for the bunch amplitudes,

$$
\frac{d a_{n}(\tau)}{d \tau}=\frac{i \lambda}{2 Q_{y}} \frac{1}{N_{\text {ion }}} \sum_{i=1}^{N_{\text {ion }}} A_{i, n}(\tau) \Phi\left[\sum_{m}\left|A_{i, m}\right|^{2}, r\right] .
$$

The factor $J_{n}=\left|A_{n}\right|^{2}$ is proportional to the action variable of the $n$th mode. We can write the argument of $\Phi$ in Eq. (A16) as the sum over the coherent modes $A_{m}$ and replace the sum over incoherent ions by the integral with the distribution function $f(J), \int d J f(J)=1$,

$$
\frac{d a_{n}(\tau)}{d \tau}=\frac{i \lambda}{2 Q_{y}} \int d J f(J) A_{n}(J) \Phi\left[J+\sum_{m}\left|A_{m}\right|^{2}, r\right] .
$$

Equations (A13) and (A17) are the basis for further study below. 


\section{Linear approximation}

In the linear approximation, $\kappa\left|A_{n}\right|^{2} \ll 1$, we can neglect the contribution of the coherent modes, assuming $A_{i, n}=$ $A_{n}\left(J_{i}\right) e^{-i \mu \tau}$ and the same time dependence for $a_{n}$. Equation (A13) relates amplitudes of ions and the beam:

$$
A_{n}(J)=-\frac{\nu_{n}}{2} \frac{a_{n}}{\mu-\left(1 / 2 \nu_{n}\right)\left[\Lambda \Phi(J, r)-\nu_{n}^{2}\right]} .
$$

Substituting that in Eqs. (A17) we get the dispersion relation for the coherent shift $\mu$. Using approximation Eq. (A8), we get

$$
\mu_{n}=\frac{\lambda \nu_{n}^{2}}{2 Q_{y}} \int \frac{d J f(J)}{\left(\nu_{n}^{2}+2 \mu \nu_{n}\right)(1+\kappa J)-\Lambda} .
$$

Here $\mu$ has to be understood as having a positive imaginary part, $\mu \rightarrow \mu+i \epsilon, \epsilon>0$. The contribution of the pole then defines the growth rate $\Gamma=\operatorname{Im}(\mu)$. For $\nu \gg \mu$,

$$
\Gamma_{n}=-\frac{\pi \lambda}{2 \kappa Q_{y}} f\left[J_{n}^{r}\right] \operatorname{Sign}[\nu],
$$

where the resonance amplitude

$$
J_{n}^{r}=\frac{\Lambda-\nu_{n}^{2}}{\kappa \nu_{n}^{2}}
$$

Resonances are possible only for $J_{n}^{r}>0$, or $\Omega_{0}^{2}>\left(\omega_{y}-\right.$ $\left.n \omega_{0}\right)^{2}$. The growth rate is positive and a mode is unstable if $\nu_{n}<0$. Hence, unstable modes are within the range $\omega_{y}<$ $n \omega_{0}<\omega_{y}+\Omega_{0}$. For the ion distribution function $f(J)$ which rolls off with $J$, the maximum growth rate is obtained for modes with the minimal $J_{n}^{r}>0$, that is for the mode $\nu_{n}^{2} \simeq \Lambda$. Therefore, the strongest mode is the mode with

$$
n \omega_{0} \simeq \omega_{y}+\Omega_{0}
$$

The latter conclusion may be sensitive to the shape of the ion distribution.

The principal value of the integral in Eqs. (A19) defines the coherent shift of a mode.

For BESSY-II parameters, there are several unstable modes. Figure 14 shows the growth rate and the coherent tune shift of these modes. In calculations, we assumed Gaussian distribution $f(J)=\left(1 / J_{o}\right) e^{-J / J_{0}}$ with $J_{0}=$ $\left(\Sigma_{y, i} / \sigma_{y}\right)^{2}=10$, and pressure 10 nTorr. Other parameters are defined in Table I.

\section{Nonlinear regime}

Equation (A17) shows what can be expected in the nonlinear regime. If the coherent amplitudes vary slowly, then Eq. (A18) would retain its form with $\Phi(J)$ being replaced by $\Phi\left(J+\left|A_{n}\right|^{2}\right)$. Respectively, the factor $(1+$
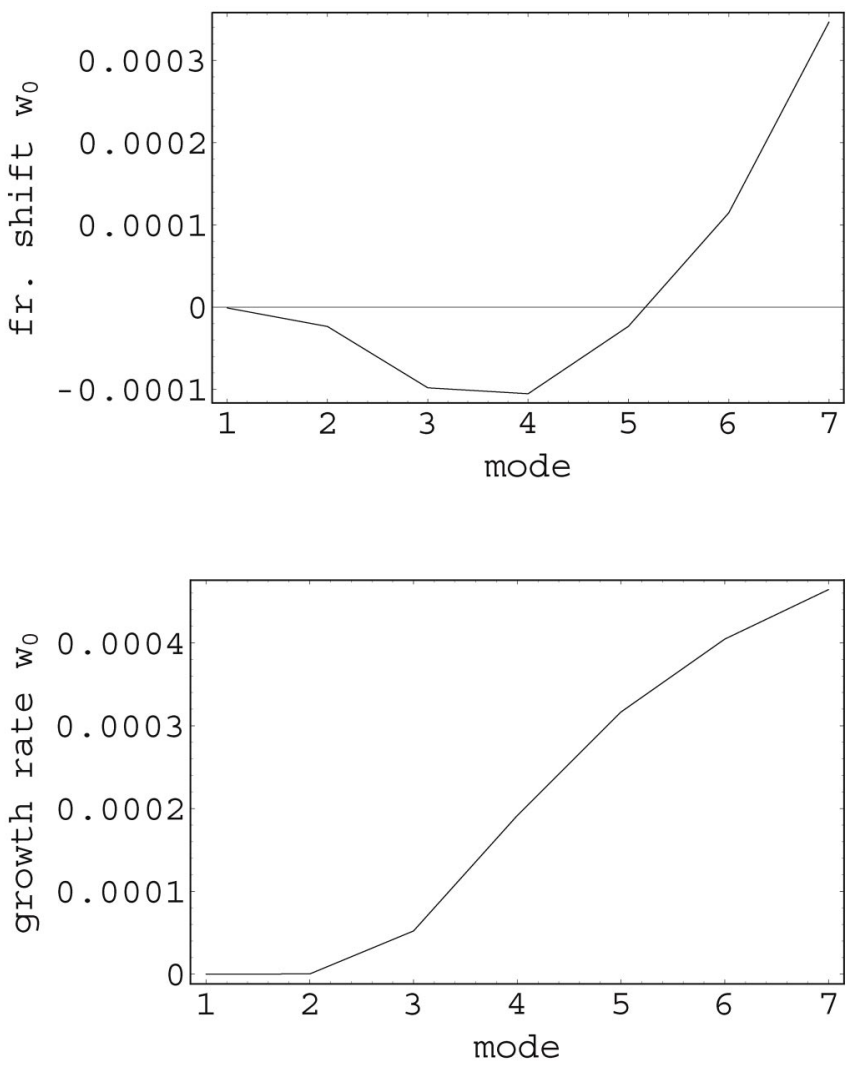

FIG. 14. Dimensionless coherent frequency $\mu / \omega_{0}$ of the beam-ion instability in the linear approximation.

$\kappa J)$ in Eq. (A19) would be replaced by $\left(1+\kappa J+\left|A_{n}\right|^{2}\right)$. The growing coherent modes $\left|A_{n}\right|^{2}$ shift the position of the resonance $J_{n}^{r}$, Eq. (A21), to

$$
J_{n}^{r}=\frac{\Lambda-\nu_{n}^{2}}{\kappa \nu_{n}^{2}}-\left|A_{n}\right|^{2} .
$$

At the same time, the instability increases the beam rms dimensions reducing $\Omega_{0}$ reducing $\Lambda$. Both factors reduce the right-hand-side in Eq. (A19) and, when it is negative, the resonance is impossible and the mode is stabilized.

For ions oscillating in the potential well of the beam, each coherent mode is equivalent to periodic perturbation with the coherent frequency $\Omega=\operatorname{Re}\left(\mu_{n}\right)$. Such a perturbation generates a separatrix where ions may be trapped. For large enough coherent amplitudes coherent modes cannot be considered independently due to overlapping of the separatrices. The ion motion becomes random and ions give random kicks to the beam. The exponential linear regime of the instability then is replaced by the diffusive linear growth of the beam amplitude with time [8].

In the extreme case of large coherent amplitudes, where $\left|A_{n}\right|^{2}$ is large compared to the incoherent spread of amplitudes, Eq. (A17) can be again simplified. Neglecting inco- 
herent $J$ in the argument of $\Phi$ and using the norm $\int d J f(J)=1$, we can reduce Eqs. (A13) and (A17) to the system of equations for the interacting coherent modes,

$$
\begin{gathered}
\frac{d a_{n}(\tau)}{d \tau}=\frac{i \lambda}{2 Q_{y}} A_{n} \Phi\left[\sum_{m}\left|A_{m}\right|^{2}, r\right] . \\
\frac{d A_{n}}{d \tau}+\frac{i}{2 \nu_{n}}\left[\Lambda \Phi\left(\left|A_{n}\right|^{2}, r\right)-\nu_{n}^{2}\right] A_{n}=\frac{i \nu_{n}}{2} a_{n} .
\end{gathered}
$$

The system can be solved numerically. We used an approximation of Eq. (A8) for $\Phi$ and 12 modes $A_{n}, a_{n}, n=$ $1, \ldots, 12$ with initial conditions $a n(0)=0.1$ and $A_{n}(0)=$ 0 . The results of calculations shown in Fig. 6 are in good agreement with tracking results.
[1] D. Teytelman, Ph.D. thesis, Stanford University (Report No. SLAC-R-633, 2003).

[2] P. Morton, in Proceedings of the Mini-Workshop on Multibunch Experiments (SLAC, Stanford, CA, 1997), pp. 565575.

[3] K. Thompson and R.D. Ruth, Phys. Rev. D 43, 3049 (1991).

[4] D. G. Koshkarev and P. R. Zenkevich, Part. Accel. 3, 1 (1972).

[5] D. Sagan and A. Temnykh, Nucl. Instrum. Methods Phys. Res., Sect. A 344, 459 (1994).

[6] K. Ohmi, Phys. Rev. E 55, 7550 (1997).

[7] T. Raubenheimer and F. Zimmermann, Phys. Rev. E 52, 5487 (1995)

[8] S. Heifets, SLAC Technical Report No. SLAC-PUB-7734, 1998. 